\title{
FIREBALL: The Faint Intergalactic medium Redshifted Emission Balloon -- Overview and 1st Science Flight Results
}

\author{
Bruno Milliard $^{\mathrm{a}}$, D. Christopher Martin ${ }^{\mathrm{b}}$, David Schiminovich ${ }^{\mathrm{c}}$, Jean Evrard ${ }^{\mathrm{d}}$, Matt \\ Matuszewski $^{\mathrm{b}}$, Shahinur Rahman ${ }^{\mathrm{b}}$, Sarah Tuttle ${ }^{\mathrm{c}}$, Ryan McLean ${ }^{\mathrm{b}}$, Jean-Michel Deharveng ${ }^{\mathrm{a}}$, \\ Frederi Mirc ${ }^{\mathrm{d}}$, Robert Grange ${ }^{\mathrm{a}}$, and Robert Chave ${ }^{\mathrm{e}}$ \\ ${ }^{a}$ Laboratoire d'Astrophysique de Marseille, 13388 Marseille CEDEX 13, France; \\ ${ }^{\mathrm{b}}$ California Institute of Technology, Cahill Center for Astrophysics, 1216 E. California Blvd., \\ Pasadena, CA 91125 USA \\ ${ }^{c}$ Columbia University Astronomy Department, 550 W. 120th St., New York, NY 10027, USA; \\ ${ }^{\mathrm{d}}$ Centre National d'Etudes Spatiales, 31401 Toulouse CEDEX 4, France; \\ ${ }^{\mathrm{e}}$ Robert Chave Applied Physics Inc., P.O. Box 266, Altadena, CA 91003, USA
}

\begin{abstract}
FIREBALL (the Faint Intergalactic Redshifted Emission Balloon) is a balloon-borne 1m telescope coupled to an ultraviolet fiber-fed spectrograph. FIREBALL is designed to study the faint and diffuse emission of the intergalactic medium, until now detected primarily in absorption. FIREBALL is a path finding mission to test new technology and make new constraints on the temperature and density of this gas. We report on the first successful science flight of FIREBALL, in June 2009, which proved every aspect of the complex instrument performance, and provided the strongest measurements and constraints on IGM emission available from any instrument.
\end{abstract}

Keywords: Instrumentation, Integral Field Spectroscopy, Ultraviolet

\section{INTRODUCTION}

We have developed and successfully flown the Faint Intergalactic-medium Redshifted Emission Balloon (FIREBALL), designed to discover and map faint emission from the Intergalactic Medium (IGM). Emission from

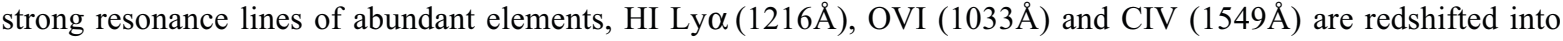
the 1950-2250 $\AA$ stratospheric balloon window at redshifts of $\mathrm{z}(\mathrm{Ly} \alpha) \sim 0.7, \mathrm{z}(\mathrm{OVI}) \sim 1.0$, and $\mathrm{z}(\mathrm{CIV}) \sim 0.3$. FIREBALL observes regions of the cosmos with known large-scale structures (traced by galaxy redshift surveys, QSO emission and absorption lines) to search for and eventually map emission from the IGM surrounding and delineating these structures.

FIREBALL, shown in Figure 1, combines a number of innovations:

- 1 meter class UV telescope, low cost optics enabled by innovative mounts ${ }^{[1]}$

- First ever integral field UV spectrograph and UV fiber optic system

- Arcsecond quality balloon pointing system, developed from scratch

- Partnership of two national space agencies

- Highly leveraged NASA resources

- 3 graduate student Ph.D.s already nearing (one is done) completion based on first two flights; 3 more graduate student Ph.D. theses in proposed time period.

- A telescope platform for testing future UV-VIS mission-enabling technologies, one of which is the planned FIREBALL Gen-2 UV-CCD detector

Space Telescopes and Instrumentation 2010: Ultraviolet to Gamma Ray,

edited by Monique Arnaud, Stephen S. Murray, Tadayuki Takahashi, Proc. of SPIE Vol. 7732,

$773205 \cdot$ ? 2010 SPIE · CCC code: 0277-786X/10/\$18 - doi: $10.1117 / 12.857850$

Proc. of SPIE Vol. 7732 773205-1 
FIREBALL's first flight occurred in July 2007. A large launch angle severed the cable powering the azimuth control mechanism, so stable pointing was not possible, but the flight was otherwise a successful engineering demonstration. FIREBALL was refurbished and modified in preparation for a second turnaround flight in the Spring of 2009 .

Our successful science flight on June 8, 2009, proved every aspect of the complex instrument performance, and provided the strongest measurements and constraints on IGM emission available from any instrument.
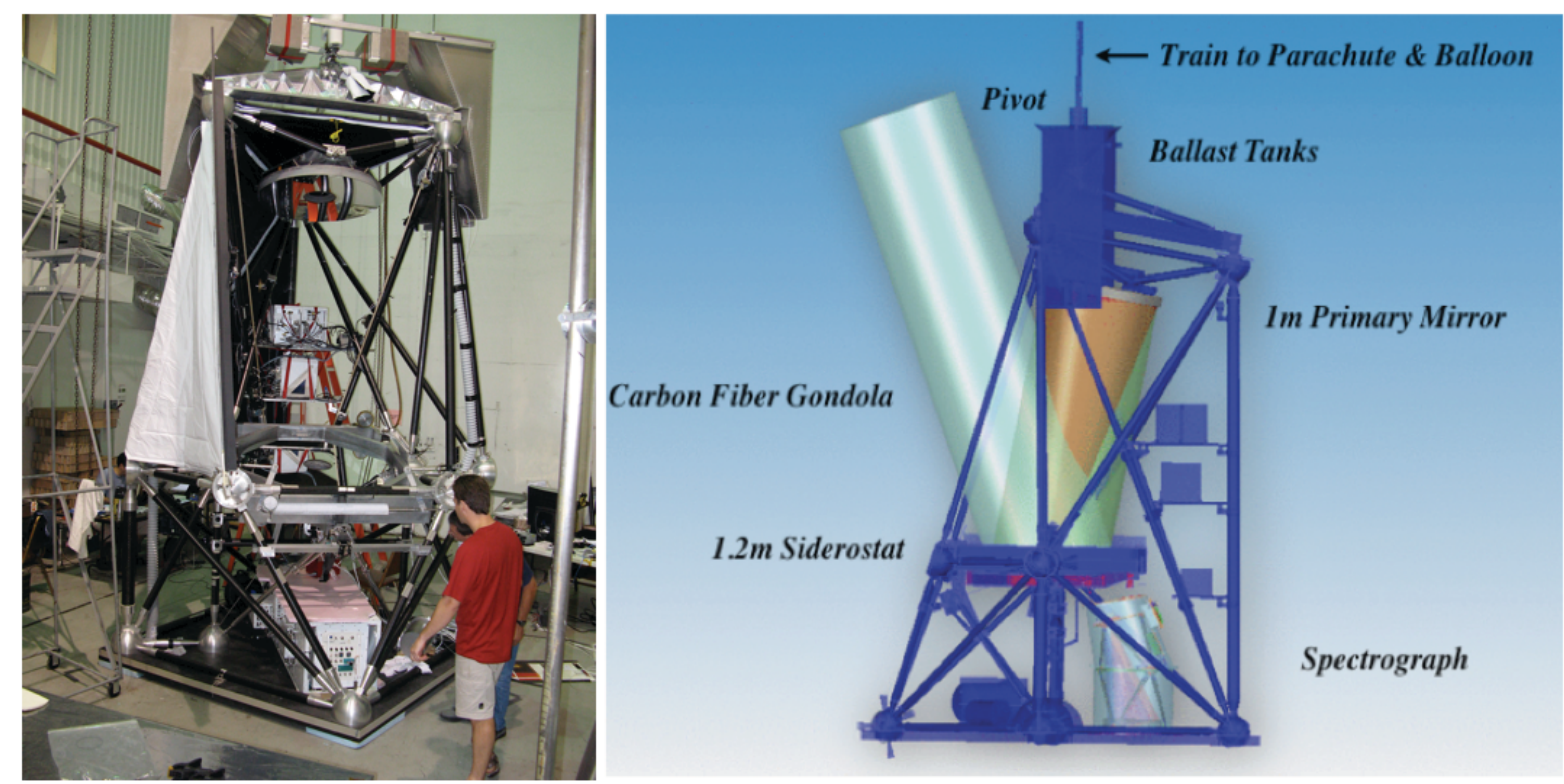

Figure 1. FIREBALL. Left: Photo during integration, Right: schematic layout showing main components.

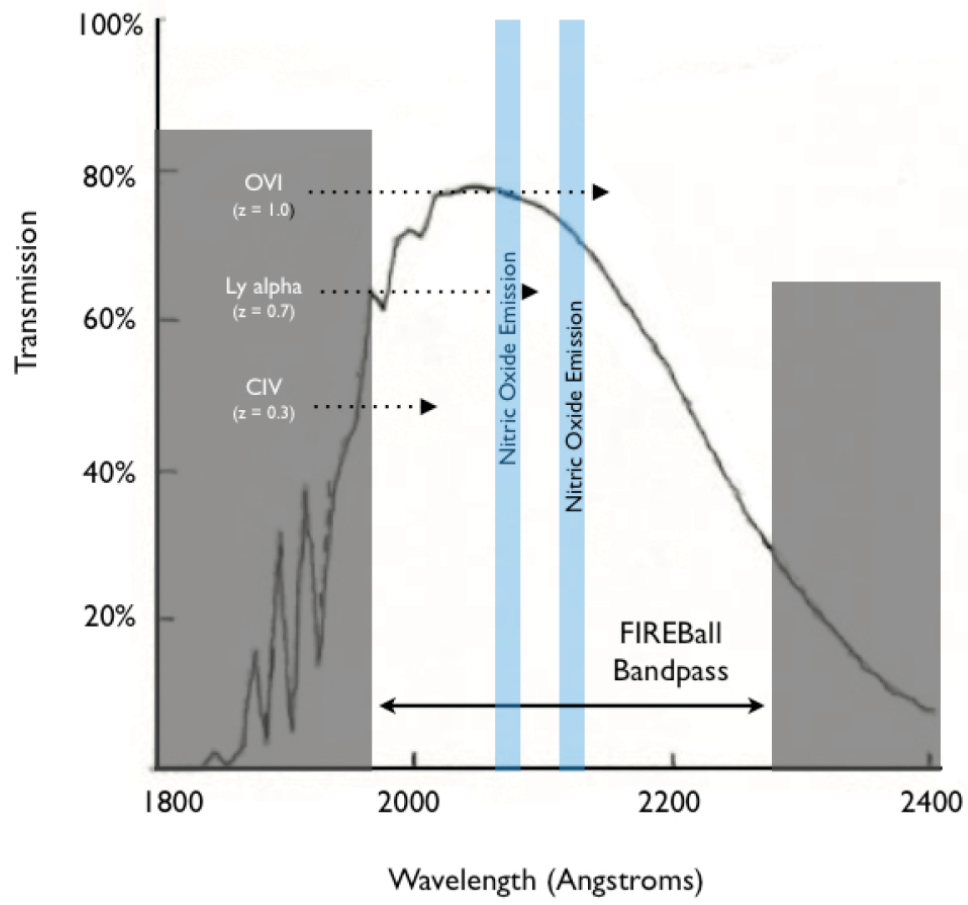

Figure 2. Balloon window in Near UV assuming 3 mbar ( $130 \mathrm{kft})$ altitude. FIREBALL altitude was $113 \mathrm{kft}$., with peak transmission of $\sim 25 \%$. Diagram shows 3 strong UV resonance lines and their corresponding redshifts in the balloon UV window. 


\section{PAYLOAD DESCRIPTION}

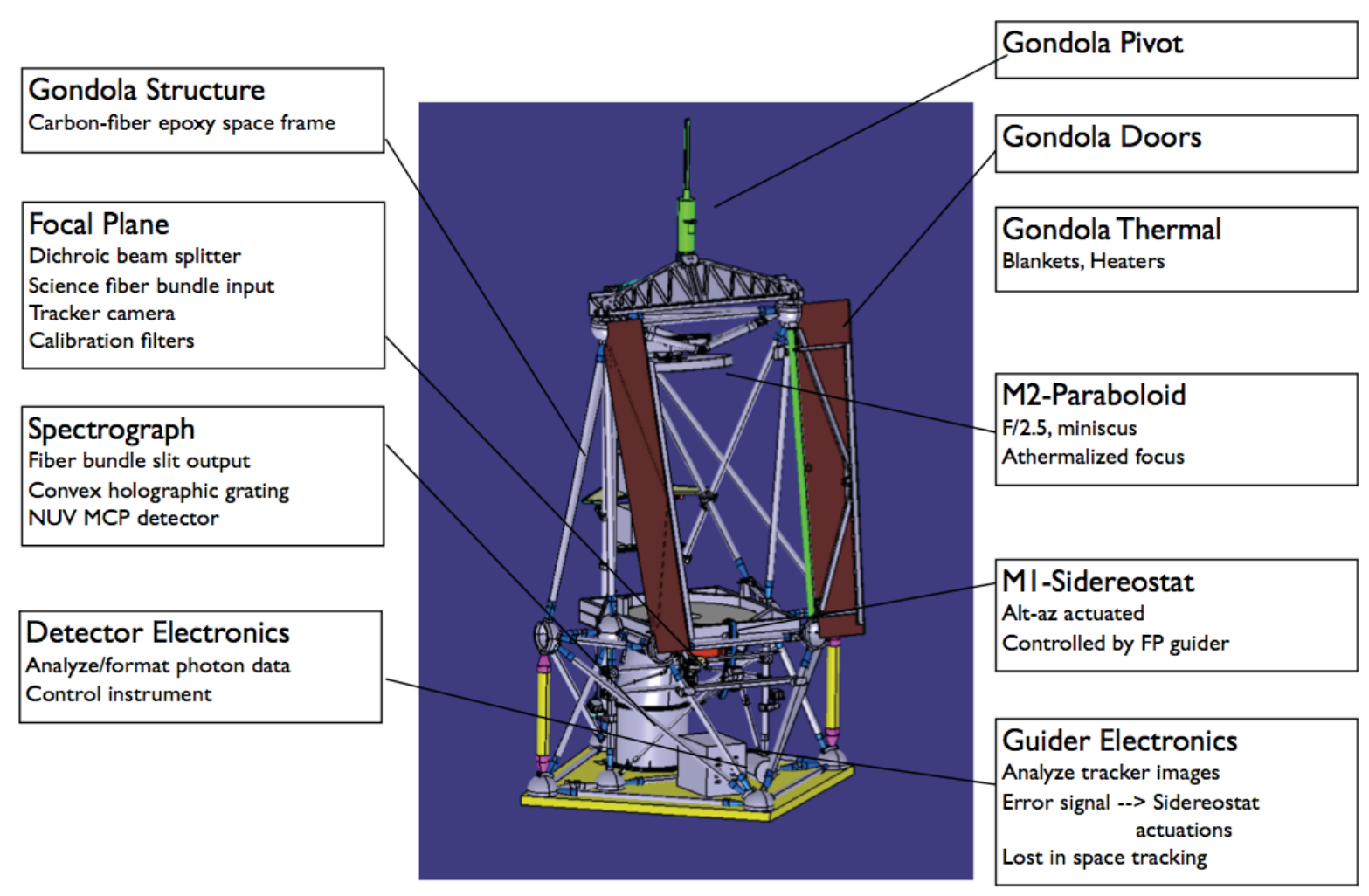

Figure 3. FIREBALL schematic layout showing main components.

Table 1 -- FIREBALL Summary

\begin{tabular}{|l|l|l|}
\hline Parameter & Value & Rationale \\
\hline Telescope Diameter & 1.0 meter (collecting area) & Required for faint emission regions \\
\hline Telescope Design & Siderostat --> Fixed f/2.5 paraboloid & $\begin{array}{l}\text { Pointing system drives smaller mass of } \\
\text { sidereostat, lower cost and mass. }\end{array}$ \\
\hline Wavelength range & $2000--2200 \AA$ & Balloon window \\
\hline Spectral resolution & R $\sim 500,0.4 \AA$ & $\begin{array}{l}\text { Maximize emission-line contrast over } \\
\text { background, resolve kinematics }\end{array}$ \\
\hline Field of view & 3 arcmin diameter & Maximize cosmic volume viewed \\
\hline Spatial resolution & $\sim 10$ arcsec & $\begin{array}{l}\text { Separate emission from galaxies, other } \\
\text { foreground objects. }\end{array}$ \\
\hline Number of IFU Fibers & 281 (science), 37 (calibration) & Derived from FOV and spatial resolution \\
\hline Detector & $\begin{array}{l}\text { GALEX Near UV spare, CsTe } \\
\text { Microchannel Plate with cross-strip } \\
\text { delay line anode, sealed tube. }\end{array}$ & $\begin{array}{l}\text { Photon-counting, low background noise, flight- } \\
\text { proven }\end{array}$ \\
\hline Sensitivity & $\begin{array}{l}\text { 30,000 LU (direct), 3000 LU } \\
\text { (statistical) }[6 \text { hours] }\end{array}$ & $\begin{array}{l}\text { Sufficient to detect bright regions and constrain } \\
\text { IGM emission. Gen-2 10x improvement. }\end{array}$ \\
\hline $\begin{array}{l}\text { Gondola/Fine Pointing } \\
\text { System Control }\end{array}$ & $\begin{array}{l}\text { Control: } 5 \text { arcsec } \\
\text { Knowledge: } 3 \text { arcsec }\end{array}$ & $\begin{array}{l}\text { Efficient observations } \\
\text { Derived from spatial resolution requirement }\end{array}$ \\
\hline
\end{tabular}

FIREBALL is a balloon borne experiment designed to observe Ly $\alpha$ emission from the intergalactic medium. It is a $1 \mathrm{~m}$ class telescope equipped with a fiber-fed UV integral field spectrograph. The integral field unit is built from $281 \mathrm{UV}$ optimized fibers and covers a hexagonal field of view 2.3 arcmin across. It feeds into an $\mathrm{R} \sim 5000$ modified Offner design spectrograph. The details of the spectrograph design are discussed in Tuttle et al. ${ }^{[2]}$ The FIREBALL detector is a legacy 
GALEX NUV microchannel plate (MCP) device ${ }^{[3]}$. The instrument exploits a narrow stratospheric balloon altitude observational window from 1950 to $2300 \AA$. The FIREBALL pointing system, discussed in Matuszewski et al. ${ }^{[4]}$ compensates for environmental perturbations during the flight and achieves 7" RMS tracking. The data capture and analysis is described in Rahman et al. ${ }^{[5]}$ FIREBALL is summarized in Table 1 and Figures 1 and 3.

The optical layout of FIREBALL is shown in Figures 1 and 3. A siderostat conveys the light to a single parabolic f/2.5 mirror. The focused beam strikes a dichroic beam splitter, which reflects light of the primary wavelength to the highresolution spectrograph. The balance of the light (visible) goes to a guider described below. Two calibration lamps $\left(\mathrm{PtNe}, \mathrm{D}_{2}\right)$ inject light into the system via the dichroic backside, with line spectra and flat field illumination using a diffuser.

Telescope. We fabricated a 1 meter telescope with excellent performance at extremely low cost, within the scope of a suborbital program by using an innovative thin mirror/hierarchical-kinematic support and telescope pointing control. The telescope consists of a 1.2-meter plano siderostat, situated on a dual axis pivot assembly, feeding a fixed 1.0-meter f/2.5 paraboloid. The siderostat provides all elevation control, over a range of 40-70 degrees, as well as fine cross-elevation control. Coarse cross-elevation is provided by the gondola/balloon pivot mechanism. Our imaging requirement is $\sim 3$ arcsec. The large mirrors have been fabricated from relatively thin (15:1) cylindrical low thermal expansion Corning TSG blanks by Optical Mechanics. We support the mirrors using an innovative 18-point configuration which limit the figure deflection to 2 microns for a contribution of $<1$ arcsecond to the image size. The mirrors were coated with an Al$\mathrm{MgF}_{2} \mathrm{UV}$ optimized coating at the GSFC coating lab.

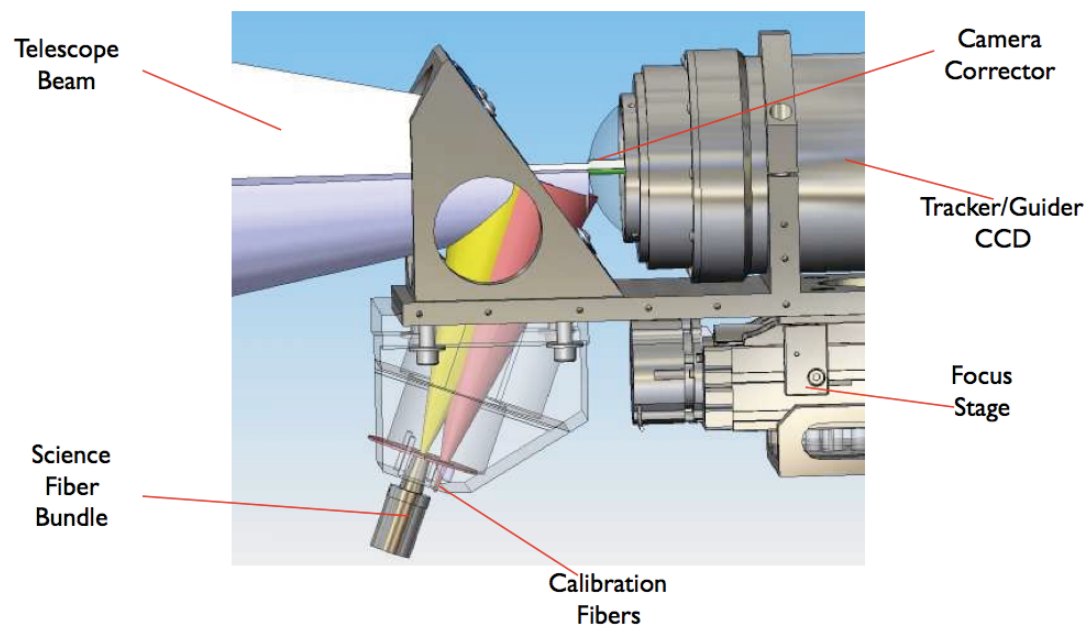

Figure 4. Focal plane layout. f/2.5 beam travels from fixed paraboloid to dichroic beam splitter. Visible light is transmitted to fine guidance camera, and 200-220 $\mathrm{nm}$ is reflected into science fiber bundle.

Dichroic Beam-Splitter and Red Blocker. An all-dielectric, broadband, red-blocking dichroic filter has been deposited on a fused silica folding flat in order to split the telescope beam between the guider camera (transmission at long wavelength) and spectrograph (reflection at shorter wavelengths). The focal plane configuration is shown in Figure 4. The red blocking coating is very similar to the design used for the NUV channel in GALEX, which achieved $>90 \%$ reflectance at $2200 \AA$ and dropping to $<10 \%$ above $3000 \AA$.

Integral Field Unit. The FIREBALL-1 IFU (Figure 5) consists of 300 UV grade (high-OH) fused silica optical fibers jacketed in polyimide. Each fiber has a core diameter of 100 microns $(8 \mathrm{arcsec})$. The fibers are mapped onto the sky in a $2.5^{\prime} \times 2.5^{\prime}$ area in the focal plane with the central fibers (on the sky) at the center of the spectrograph slit, and the edge fibers (on the sky) towards the outer edge of the slit. The fiber focal ratio (f/2) is a good match to the telescope and Offner spectrograph focal ratio of $\mathrm{f} / 2.5$. The high speed of the system minimizes focal ratio degradation. With a fiber length $<0.5 \mathrm{~m}$, we obtain a transmission of $70 \%$. The highly modular IFU design allows us to readily adapt our focal plane geometry and spectrograph slit filling factor to optimize our mapping strategy for FIREBALL-2. 


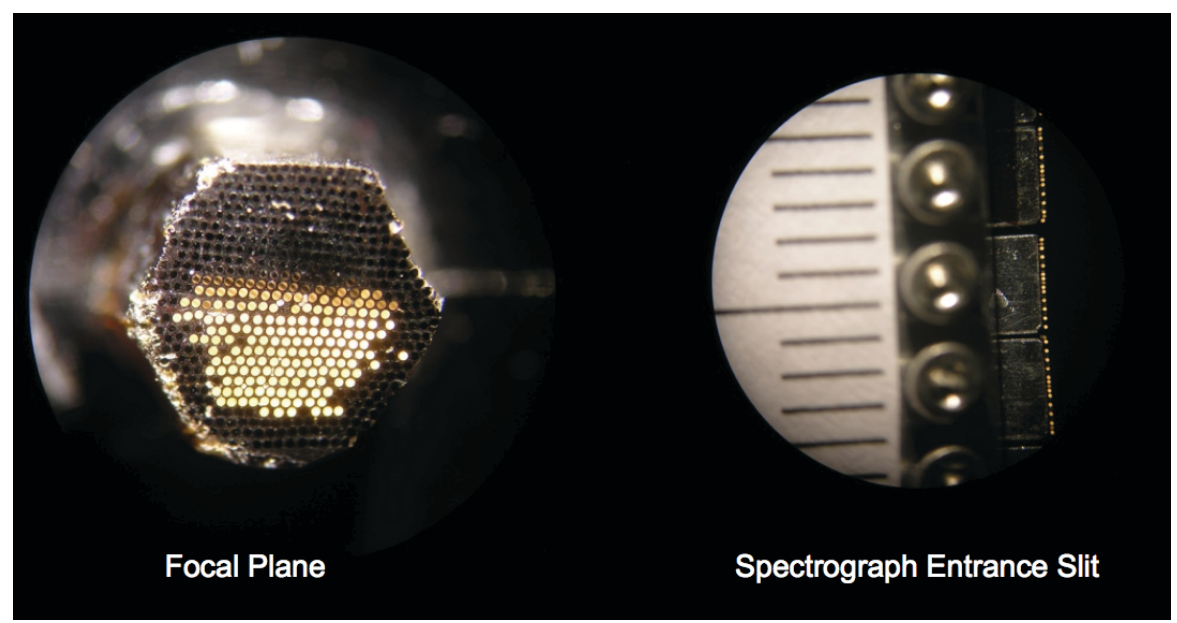

Figure 5. FIREBALL Integral Field Unit. LEFT: fiber bundle mounted at focal plane. RIGHT: reformatted fibers at entrance slit of spectrometer.
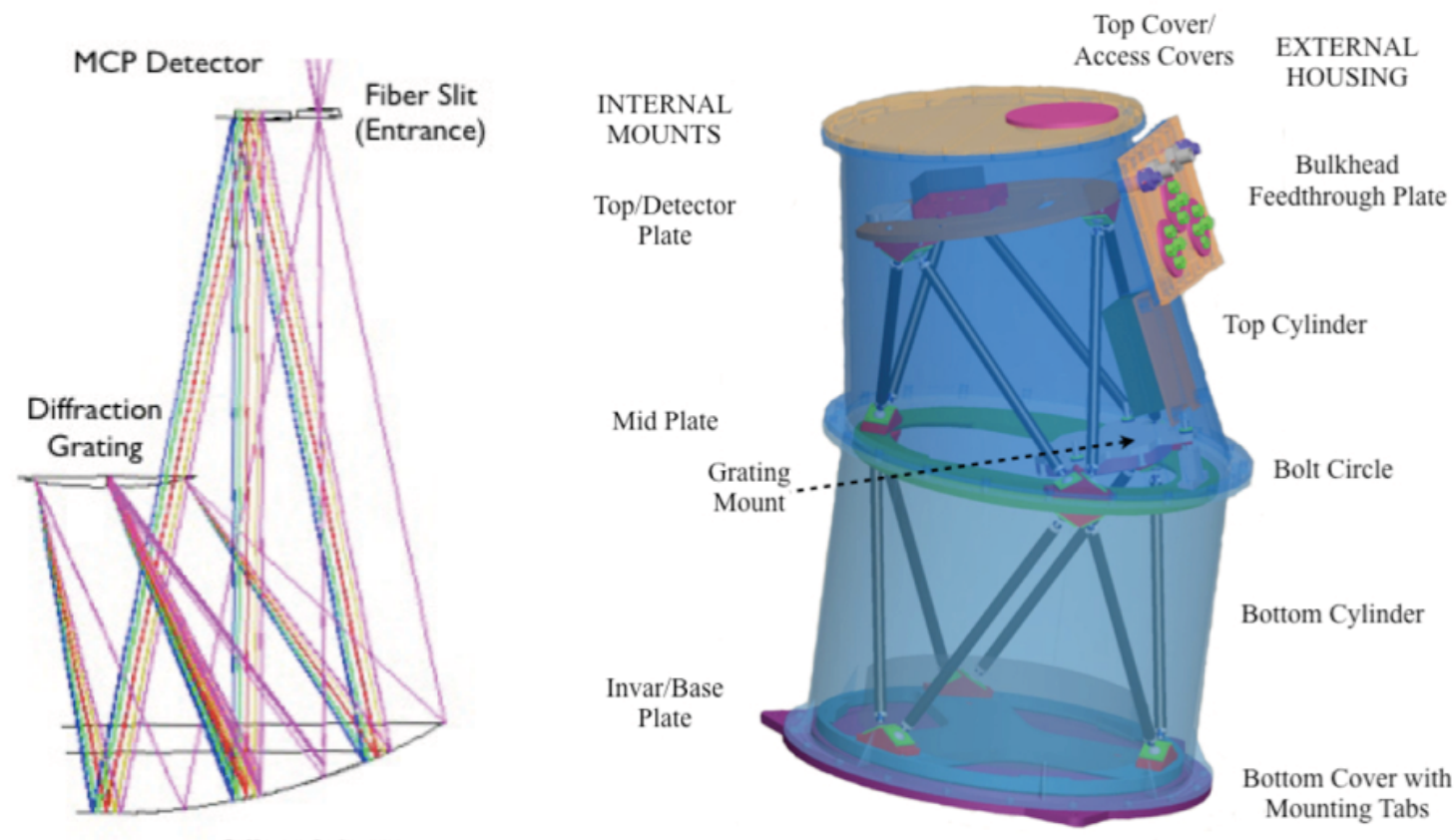

Offner Sphere

Figure 6. Spectrograph layout. LEFT: optical raytrace. RIGHT: mechanical layout.

Spectrograph. The spectrograph, illustrated in Figure 6, is a modified Offner design, with a spherical mirror (radius of curvature $876 \mathrm{~mm}$, diameter $480 \mathrm{~mm}$ ) and concentric convex spherical grating (radius of curvature $470 \mathrm{~mm}$ ). Light diverging from the exit of the fiber bundle, configured as a slit, strikes the spherical mirror. Converging light is then diffracted by the $230 \mathrm{~mm}$ diameter, 4800 groove $/ \mathrm{mm}$ grating. Finally, a diffracted diverging beam strikes the sphere again, and is focused onto the detector. This design provides good resolution and imaging performance over the long slit, an all-reflective configuration, and a compact package. The grating, provided as a contribution by CNES and LAM, has holographically ruled, straight grooves, manufactured by Jobin-Yvon. Jobin-Yvon has a long history of providing 
excellent UV diffractive optics to NASA missions such as FUSE and GALEX. Grating efficiency has been measured at $\sim 38 \%$, near theoretical limits.

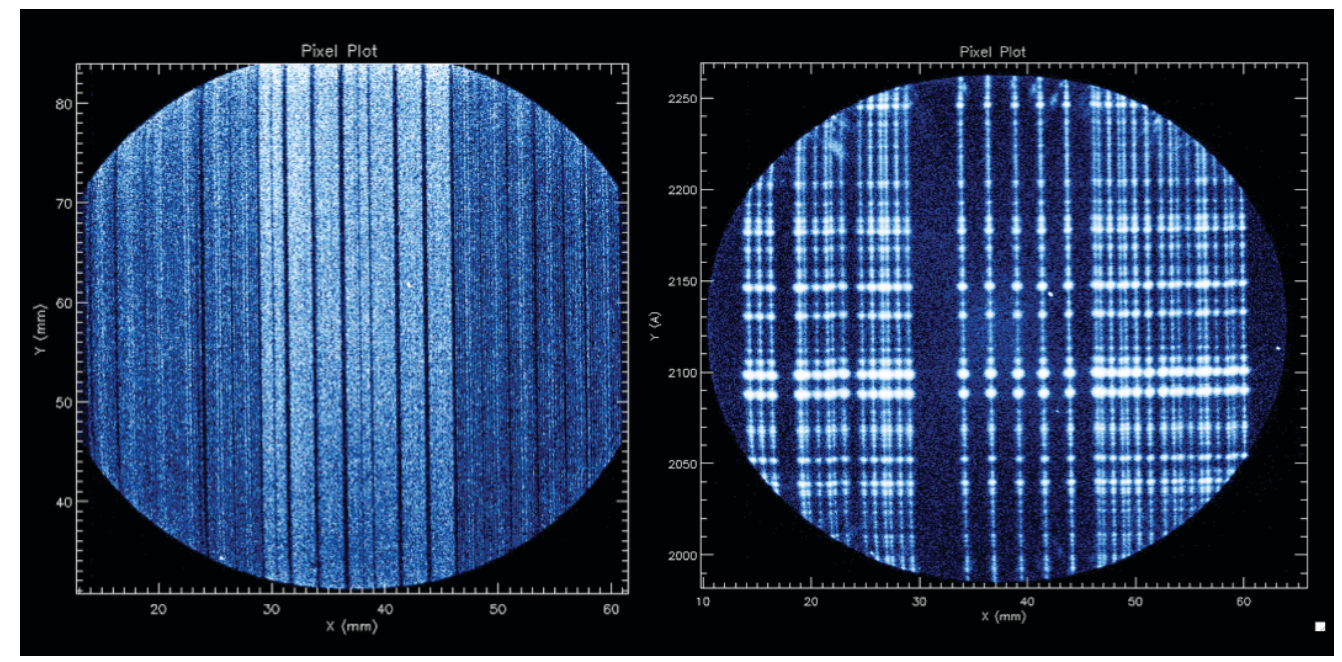

Figure 7. LEFT: continuum lamp illuminating flat field diffuser. RIGHT: Spectrograph detector image of Arc lamp illuminating calibration fibers.

The optical interface through a flexible fiber enables a very simple spectrograph-mounting scheme with the optical bench fixed with respect to the gondola structure. This mechanical decoupling from the telescope and siderostat eliminates time dependent flexure. Spectrograph optics are mounted on an athermalized hexapod that provides the mechanical interface between the Offner sphere, grating and slit/detector assembly. In-flight thermal control (to $\Delta \mathrm{T}+/-5$ C) further minimizes ground-flight changes to the optical train. The FIREBALL modular, light-sealed spectrograph housing forms a pressure vessel that maintains the Gen-1 HV detector at safe pressures (far from the coronal discharge regime).

Calibration System. A flight calibration system produces arc line and continuum spectra in science and calibration fibers (Figure 7). Either arc line or $\mathrm{D}_{2}$ continuum lamps can illuminate calibration fibers which are interleved in the fiber bundle and spectrograph entrance slit. Arc line spectra were taken periodically throughout the flights to correct for any wavelength drifts. Arc and continuum lamps can also illuminate a diffuser at the focal plane, and when the telescope is placed in autocollimation the entire fiber bundle is illuminated for calibration. Additionally, on the ground a star simulator was used to measure image quality and map the fiber bundles.

Gen-1 Detector. FIREBALL-1 uses an existing GALEX spare Near UV sealed-tube, microchannel plate detector. The $\mathrm{QE}$ at $2100 \AA$ is $\sim 5 \%$, field of view is $65 \mathrm{~mm}$, spatial resolution is $\sim 60$ microns (FWHM). Dark background is $\sim 1 \mathrm{ct}$ $\mathrm{cm}^{-2} \mathrm{~s}^{-1}$, which currently limits our sensitivity.

Gondola. As part of the FIREBALL collaboration, Laboratoire D'Astrophysique de Marseille (LAM) and CNES have constructed a state-of-the art, arcsecond quality pointing gondola. LAM brings both extensive balloon flight experience and tested equipment to the collaboration. The gondola is the mechanical structure, the optical bench, and the tracking framework for the experiment. The design is a sparse set of nodes connected with carbon fiber rods, forming a stiff (first resonance above $20 \mathrm{~Hz}$ ), thermally-stable kinematic structure. Optical mounts to the gondola have been athermalized to compensate for residual expansion effects in the rods and the aluminum couplings. The focal plane includes a stage for flight focus adjustments.

Pointing and Tracking. For FIREBALL the pointing requirement is to maintain a consistent attitude throughout the observation for exposure uniformity. The FIREBALL design adapts the successful FOCA gondola design, which delivered an in-flight pointing jitter of $2 \operatorname{arcsec}(\mathrm{rms})$. The design uses a two-stage tracking concept. The housing of the gondola is passively and actively damped and is stabilized azimuthally with a motorized suspension bearing to provide a reference platform for the second stage tracking. Gyros on the gondola provide velocity reference and a two-axis magnetometer measures azimuth. The motorized bearing is torque controlled and provides a viscous friction that damps torsional oscillations of the balloon suspension. Pendulum oscillations of the gondola platform are damped by pendula 
immersed in oil and 2 inertial wheels. With these controls the gondola achieves 2-4 arcmin stability. The second stage controls the telescope and instrument with elevation and cross-elevation motors. The motor drive signal takes input from three sources: (1) Gyro sensors on the gondola feed forward the rate of large velocity perturbations at the gondola; (2) A wide-field multi star sensor that views the siderostat is used as the primary tracking reference; and (3) Final fine corrections to the motor output come from the CCD star sensor at the focal plane. Field rotation can be nulled over minute timescales using a combination of azimuth and siderostat cross-elevation.

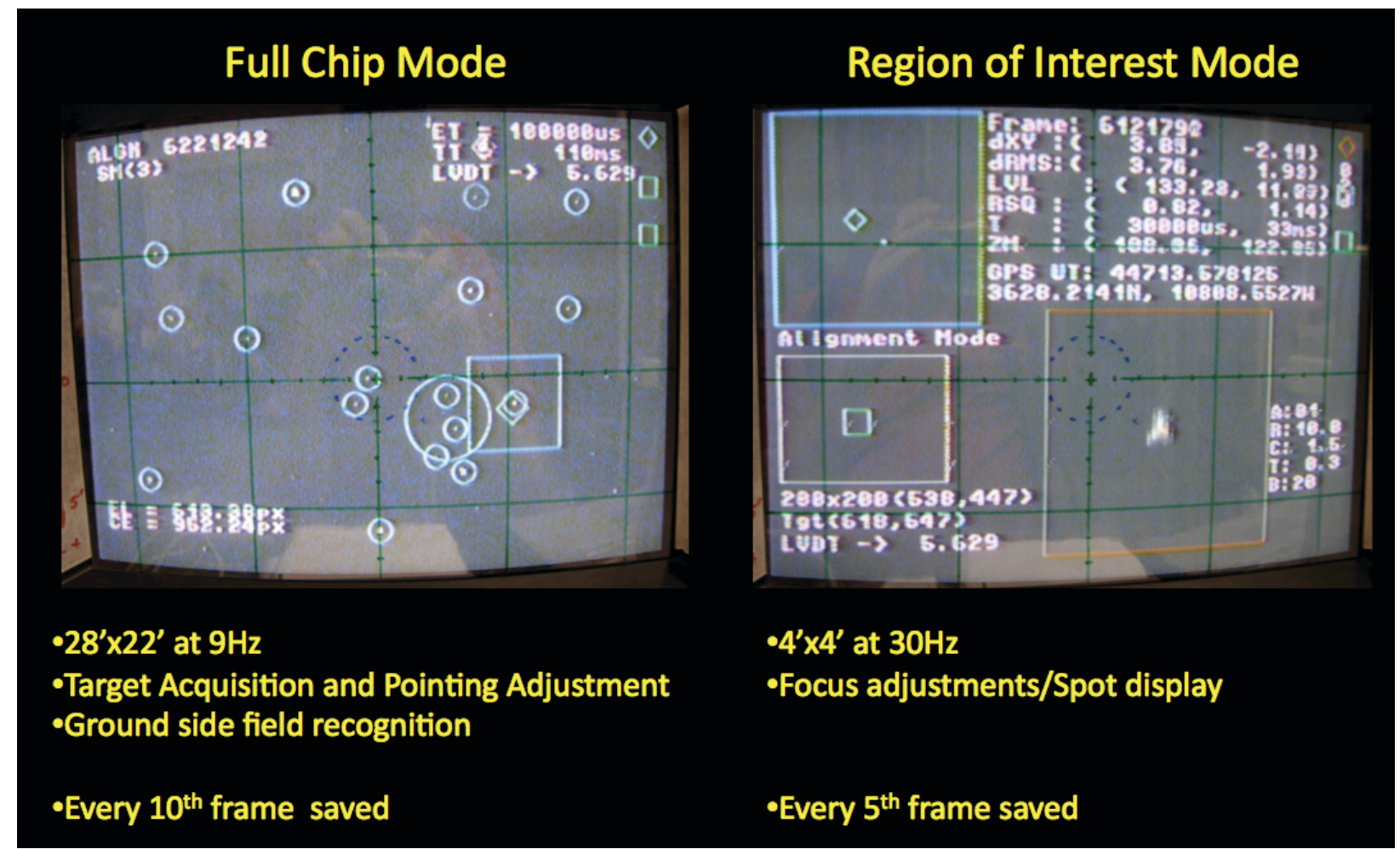

Figure 8. Guider field, Flight\#2. Guide star is centered in diamond, circle is IFU field of view. Guider field of view is $22^{\prime} \times 28^{\prime}$.

During Flight\#2 the gondola coarse target acquisition and pointing system worked well, bringing us within $\sim 10-20$ arcminutes of each target. The guide camera system was then deployed to acquire the target into the IFU field of view, to maintain fine pointing, and to execute dither patterns to improve exposure uniformity. A sample screen shot is shown in Figure 8. Our typical rms pointing stability was 3 arcsec in the elevation direction, and 7 arcsec in the somewhat noisier cross-elevation axis.

Technical Performance. Fortunately every aspect of the FIREBALL system performed flawlessly during Flight\#2. We obtained a full night of observations, including three science targets and two alignment/calibration stars. Image quality during the flight was within specifications, $\sim 2-3$ arcseconds. Focus showed good stability and required only a single adjustment. The estimated end-to-end efficiency is $\sim 0.1 \%$, including a contribution from the atmosphere of $25 \%$. This is based on the calibration star and target QSO. Sky background included three well known NO bands at 1985, 2061, and 2141 $\AA$. The detector background, which was identical to ground levels, dominates the sky background even in the lines. The low level of the airglow $(\sim 10,000 \mathrm{LU}$ in the restricted bands, $<1000 \mathrm{LU}$ outside these bands) is extremely encouraging for future flights of FIREBALL Gen-2.

\section{FLIGHT RESULTS}

FIREBALL was launched for the first time on July 22, 2007 from Palestine, Texas. The flight was an engineering success and verified all subsystem performance except for the azimuth control mechanism. Inspection and analysis following recovery showed that the cable connecting the mechanism to the control and power in gondola was severed at 
launch due to an unexpectedly large launch angle. During the flight we were able to successfully test the fine guidance system on passing bright stars, and test the UV throughput. FIREBALL was recovered after a hard landing. The primary mirror had broken loose from its kinematic mounts, but was captured by a safety line. The damage required regrinding the back, stripping and recoating the surface (at GSFC), and bonding new mounting hardware. Additional modifications were made to the payload for Flight \#2, including a new fiber bundle, better spectrograph focus and pre-flight calibration, improvements to the guiding system, streamlining the command system, and improving the performance of the gondola pointing control system.

FIREBALL was launched on June 8, 2009 from Ft. Sumner, New Mexico. The goal was a turnaround flight spanning a full night at a minimum altitude of $120 \mathrm{kft}$. An unusual weather pattern of early monsoons led to weeks of poor surface winds and downrange altitude predictions that fell far below our minimum requirements. In the end we had to launch in sub-optimal conditions. The flight achieved an average altitude of $113 \mathrm{kft}$., severely degrading the atmospheric transmission in the balloon window. Fortunately every aspect of the FIREBALL system performed flawlessly as discussed above. We obtained a full night of observations, including three science targets and two alignment/calibration stars.
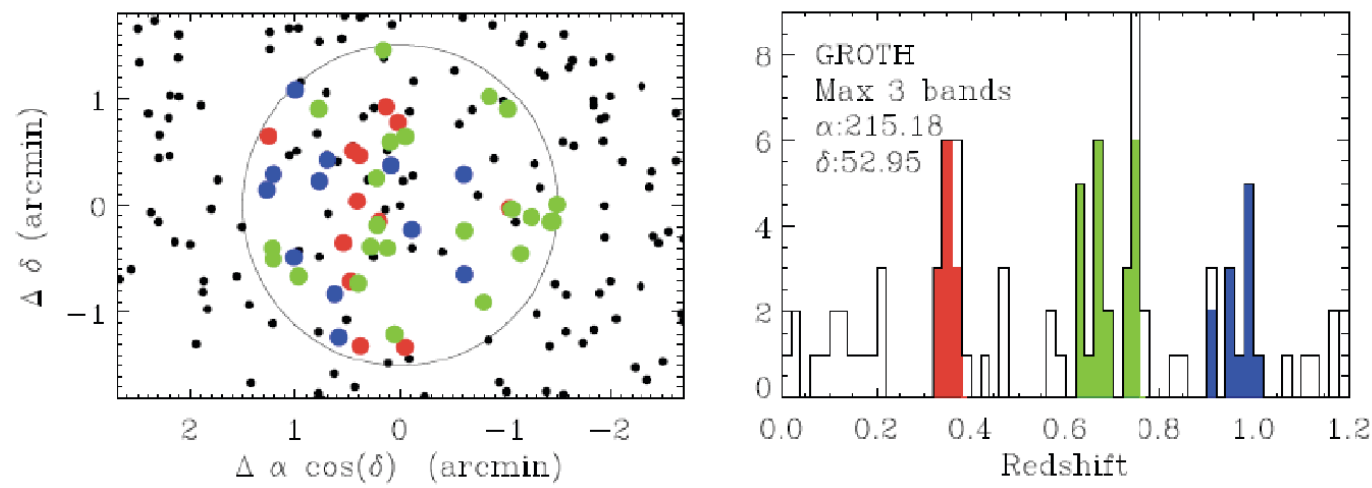

Figure 9. Redshift distribution of galaxies with potential CIV (red), Lyo (green), and OVI (blue) emission in FIREBALL band and IFU.

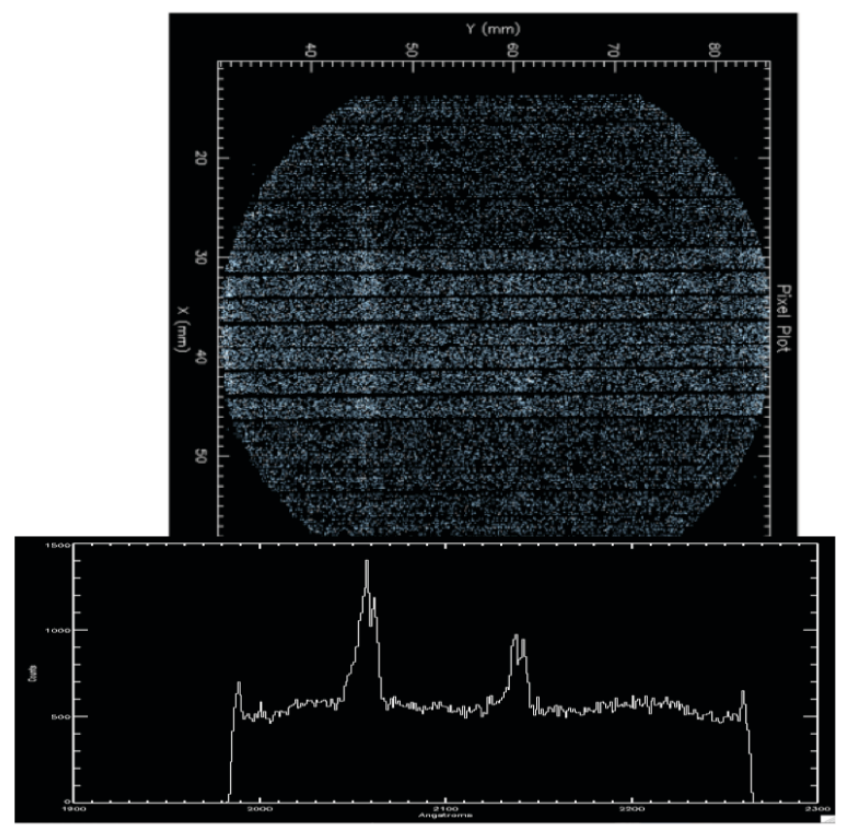

Figure 10. Flight spectrometer image showing two NO-bands. Background is dominated by detector dark counts. 
Science Targets. Science targets were selected from the following categories: 1) galaxy overdensities at the appropriate redshifts for the 3 lines of interest, 2) QSOs with absorption from the Warm-Hot IGM in the FIREBALL bandpass, 3) QSOs with Ly $\alpha$ emission redshifts in the FIREBALL bandpass, and 4) UV luminous galaxies. Flight \#2 included three science targets from categories 1) and 2). Multiple targets were chosen so that different observation and detection strategies could be explored, and to provide targets during the entire night as primary targets were eclipsed by the balloon at elevation angles exceeding 68 degrees.

Extended Groth Strip (EGS or AEGIS). A single target in this, our highest priority field, was observed over its full availability window (elevation limited by absorption and the siderostat $\left[>40^{\circ}\right]$ and balloon obscuration $\left[<68^{\circ}\right]$ ). This field is one of the most surveyed in astronomy, with extensive coverage by the DEEP2 (optical photometry and Keck redshifts $0<\mathrm{z}<1.5$ ), GALEX, Chandra, Spitzer, VLA, HST/ACS, Herschel, etc. We selected the target using the following criteria: 1) large overdensities of galaxies at $\mathrm{z} \sim 0.7$ (Ly $\alpha$ redshifted into the FIREBALL band and away from NO bands); 2) overdensities of galaxies at $\mathrm{z} \sim 1.0$ (OVI redshifted into the FIREBALL band); 3) overdensities at $\mathrm{z} \sim 0.3$ (CIV in balloon band) (see Figure 9); 4) availability of a guide star. We obtained 2.1 hours on EGS.

DEEP2 ZLE is another DEEP2 survey field selected using the same criteria as for EGS. We observed this target for 2.5 hours.

PG1718+481 is a QSO that has been observed by HST (STIS) and has an OVI absorber (as well as several Ly $\alpha$ absorbers) in the FIREBALL band. We observed this target for 1 hour.

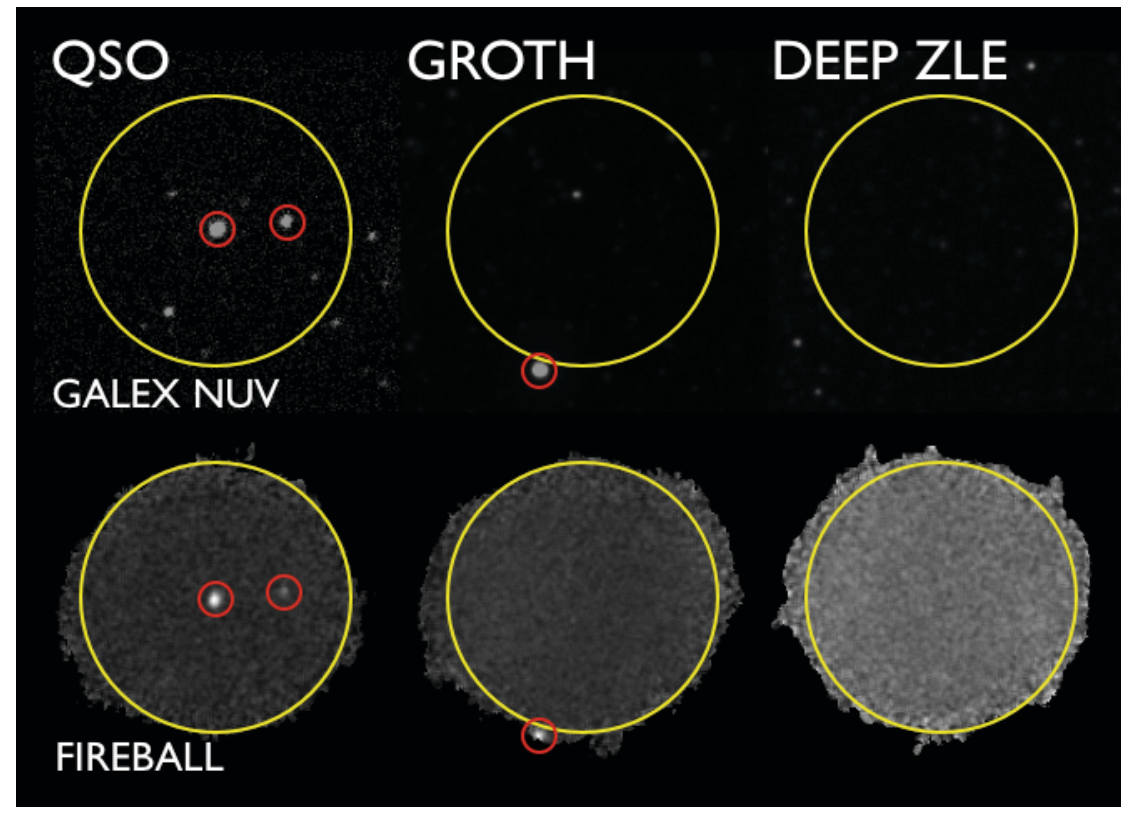

Figure 11. Reconstructed broadband "image" (3D cube collapsed in wavelength) compared to GALEX deep images of 3 science fields. These are the first reconstructed IFU images in the UV. FIREBALL already obtains limits on IGM line emission better than GALEX (image and grism) by virtue of its narrow band optimized for IGM/CGM line widths and limits imposed by source confusion in GALEX data. GALEX images are used as well to remove foregrounds and central galaxy emission.

Pipeline Data Reduction. One of the most complex tasks we have faced is reconstructing spectroscopic data cubes and photon-lists tagged by time, RA, DEC, and $\lambda$. Three data sources are merged: detector, guide camera, and gondola engineering. We have just released Version 2 photon-lists that correct for the following effects: target dither and motion (tracked by the guide camera), field rotation, detector mapping to fiber coordinates and to spectrometer wavelength, detector non-linearity, walk, wiggle, and thermal drift, fiber flat-field, detector flat-field, wavelength drift due to thermal changes (small). Steps following rectified photon-lists are detector background subtraction, accumulation of data cubes (RA, Dec, $\lambda$ ), foreground removal, source spectrum extraction for point sources, and a suite of analyses designed to detect or constrain diffuse IGM and CGM emission. Figure 10 shows the raw data in detector coordinates, and Figure 11 
shows the images obtained for all three targets compared to a GALEX image. These are the first-ever IFU-reconstructed images obtained in the ultraviolet.

Science Analysis. We employ four analysis approaches for detecting and constraining diffuse emission: 1) blind search, 2) galaxy correlations, 3) QSO correlations, and 4) filament correlations. In each, two basic approaches can be isolated: direct and statistical detection. Direct detection is the most powerful way to discover and map emission from single regions. Statistical detection can achieve far deeper sensitivity at the expense of isolating individual sources. While these are already interesting limits, we show in $\$ 4$ how these results will dramatically improve with future FIREBALL Gen-2 (Flight \#3) observations. Table 3 summarizes the observational limits; with C8 showing how these limits will improve in FIREBALL Gen-2.

1) Blind search uses optimized detection algorithms to search for, detect, and/or constrain line emission at arbitrary wavelengths, positions, and scale-sizes. There are two principal methods. The first (Blind-direct) is to search for enhancements in the $3 \mathrm{D}$ data cube in a fashion similar to that employed for 2D source detection for sources of unknown and arbitrary size and position in imaging data. The 2D algorithms are readily adapted, and can be tested on simulated data. The second method (Blind-statistical) is to measure either the Voxel Distribution Function or its power spectrum and compare that to the expected Poisson-noise power spectrum. Any IGM or CGM emission correlated with large-scale structure usually traced by galaxies will have a characteristic power spectrum (see Figure 12).

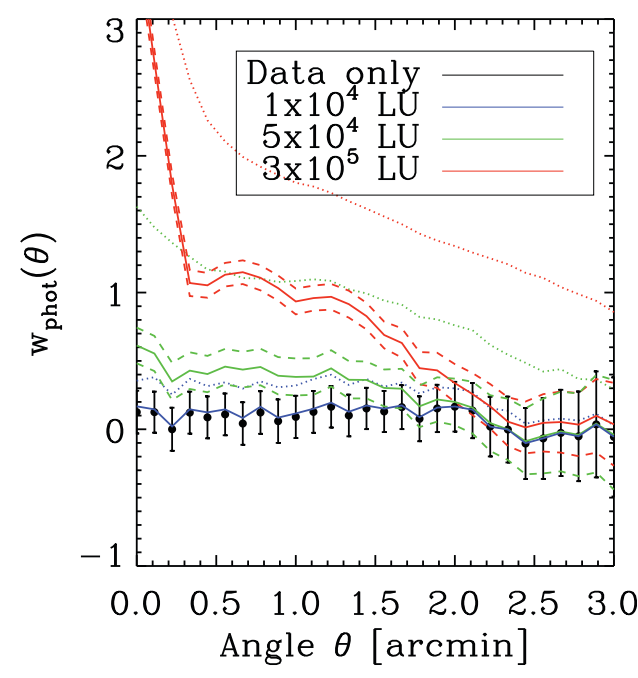

Figure 12. Constraints on IGM emission from blind statistical search using photon correlation function. Black shows FIREBall-1 w(q) from GROTH field. Red, green and blue lines show $w(q)$ when a simulated $30 " x 30 "$ source is added with different intensities. Dashed lines are $1 \mathrm{~s}$ error bars. Dotted lines show prediction for 30" filamentary source with comparable intensity. Data are consistent with no detected signal at the $\sim 20,000 \mathrm{LU}$ level.

2) Galaxy correlations. It is likely that the brightest IGM emission is correlated with galaxies. There are three approaches. Galaxy-direct examines individual galaxies for emission in a 2D "image" of the Lyo, OVI, or CIV line at the galaxy redshift, or in an azimuthally averaged radial profile. Galaxy-stacked performs the same analysis on a stack of all the galaxies for which a given line falls in the FIREBALL band, or for a subset binned by a galaxy physical parameter such as luminosity or star formation rate (SFR). Finally Galaxy-statistical performs a crosscorrelation of galaxy locations and data cube counts to search for a significant cross-correlation or cross-spectrum signal. An example of this is shown in Figure 13.

3) QSO correlations. IGM emission could be found at redshifts corresponding to the QSO systemic redshift (or nearby), or at absorption-line redshifts associated with any line species. As above, QSO-emission/absorption-direct searches for emission at the QSO systemic redshift/QSO absorption line redshifts. QSO-statistical performs a cross-correlation of either the QSO absorption-line spectrum itself or the redshift locations with the data cube.

4) Filament correlations. Galaxies are strung out on filaments of the Cosmic Web. While only deep IGM emission mapping will delineate these filaments for the first time, we can with various algorithms guess at their locations using the galaxy distribution. Filament-direct will constrain emission from individual filaments (binned along and transverse to the projected filament). Filament-stacked does the same for a stack of projected filaments.

Physical Implications of FIREBALL First Constraints. We used the above techniques to search for and constrain IGM emission. No statistically significant detections have been obtained to date. The upper limits provide the first direct constraints on models for IGM emission and associated physical conditions. We summarize our preliminary constraints on IGM emission levels in Table 2. The scientific implications of these constraints are under study, but some preliminary discussion and very rough numerical estimates are illustrative. We discuss four issues: 1) constraints on physical conditions in the IGM and CGM; 2) limits on gravitational energy dissipation in cold flows; 3) limits on mechanical energy deposition in galactic winds and feedback; 4) constraints on properties of the cosmic web. 

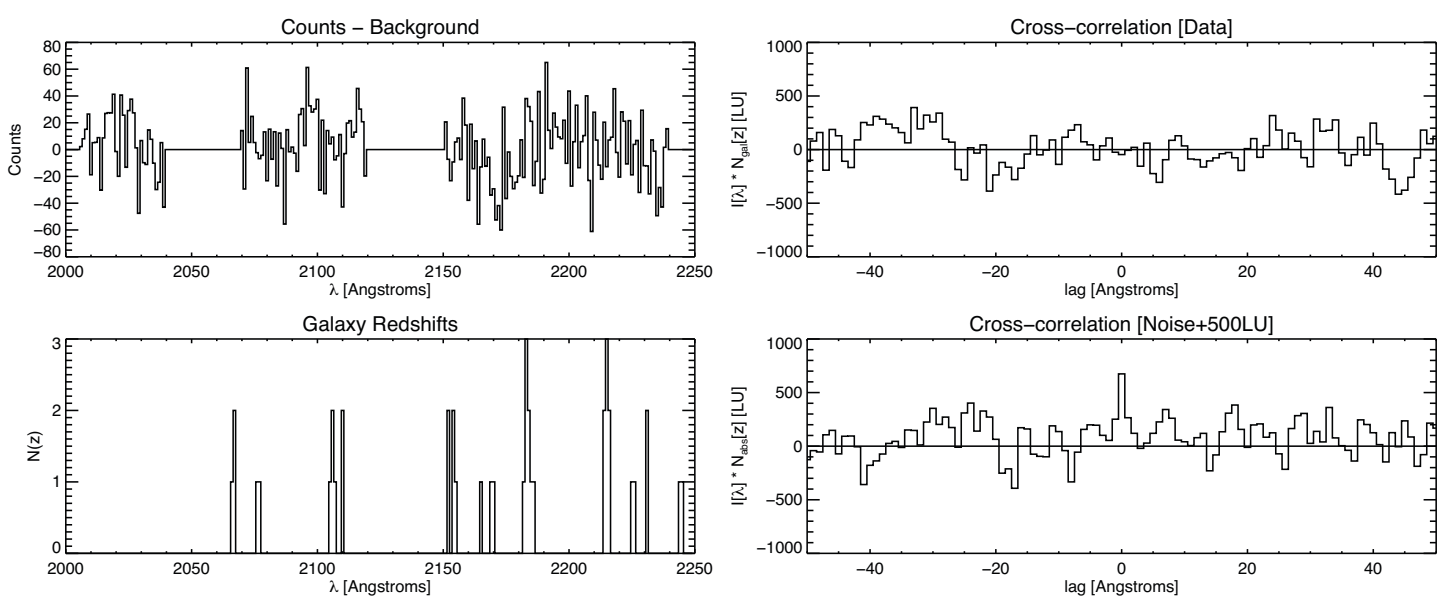

Figure 13. Ly $\alpha$ Galaxy-statistical cross-correlation shows that deep flux levels can be reached using statistical techniques. Left shows actual counts (NO bands removed) and redshift distribution. Right shows cross-correlation of left 2 vectors, and simulated data of comparable noise plus a $500 \mathrm{LU}$ emission signal with a flat distribution occupying the full IFU field of view.

Table 2 -- Preliminary Estimates of Upper limits from Flight\#2 Targets \& FB-2 Sensitivity (LU $\left.{ }^{\dagger}\right)$

\begin{tabular}{|l|l|c|c|c|c|c|c|c|}
\hline Row & Constraint & $\begin{array}{c}\text { typ. \# } \\
\text { sources }\end{array}$ & $\begin{array}{c}\text { Scale } \\
\text { (") }\end{array}$ & EGS & DEEPZLE & PG 1718 & Combined & FB-2 \\
\hline & C1 & C2 & C3 & C4 & C5 & C6 & C7 & C8 \\
\hline R1 & Integ. Time [hrs] & & & 2.1 & 2.5 & 1 & 5.6 & $2(6)$ \\
\hline R2 & Blind-direct & & 30 & 90000 & 80000 & & 55000 & $\mathbf{1 4 0 0}$ \\
\hline R3 & Blind-statistical & & 30 & 12000 & 10000 & & 7300 & $\mathbf{1 0 0}$ \\
R4 & Galaxy-direct & & 30 & 43600 & 40000 & & 26700 & $\mathbf{1 4 0 0}$ \\
\hline R5 & Galaxy-stacked & 36 & 30 & 4600 & 4200 & & 2800 & $\mathbf{2 0 0}$ \\
\hline R6 & QSO-direct & & 180 & & & 9000 & & $\mathbf{4 0 0}$ \\
\hline R7 & QSO-statistical & 36 & 180 & & & 1500 & & $\mathbf{8 0}$ \\
\hline R8 & Filament-direct & & 180 & & 4300 & & 3000 & $\mathbf{2 0 0}$ \\
\hline R9 & Filament-statistical & 38 & 180 & & 700 & & 500 & $\mathbf{4 0}$ \\
\hline
\end{tabular}

${ }^{\dagger} 1 \mathrm{LU}=1 \mathrm{ph} \mathrm{cm}^{-2} \mathrm{~s}^{-1} \mathrm{sr}^{-1}$

1) Constraints on CGM physical conditions. Models predict a wide range of CGM brightnesses with some exceeding $10,000 \mathrm{LU}[8]$ in rare locations. Models can be used to estimate the average CGM brightness over regions of $\sim 300 \mathrm{kpc}$ diameter, and for Ly $\alpha$ range from 1000-10,000 LU. The combined constraint in Table 2 [R5|C7] is a useful limit on the average CGM brightness, and we are in the process of translating this into physical constraints.

2) Limits on gravitational energy dissipation in cold flows. Numerical simulations now predict that significant IGM gas accretion in cold $\left(\sim 10^{4-5} \mathrm{~K}\right)$ flows fuels galaxy formation and on-going star formation in the early universe, perhaps extending to $\mathrm{z}=0$ [9]. Amazingly, it is estimated that $\sim 80 \%$ of the gravitational potential energy dissipated by the accreting gas may be radiated as Ly $\alpha$ ! Limits on Ly $\alpha$ therefore potentially place strong constraints on the accretion rate of new gas. Our preliminary statistical limit of $\sim 3000 \mathrm{LU}$ (Table 2 [R5|C7]) corresponds to a limit for cold flows into an average dark-matter halo at $\mathrm{z} \sim 0.7$ of $M_{D M H} \dot{M}_{\text {bary }}<10^{13} M_{\odot}^{2} y r^{-1}$ Future analysis will segregate these limits by halo mass since lower mass halos (more numerous) are likely to be fed by cold flows late in cosmic history ${ }^{[7]}$.

3) Limits on mechanical energy deposition from feedback. It is not known what fraction of starburst wind energy is radiated as Ly $\alpha$, although limits at high redshift would suggest $<10-30 \%$. Based on scaling star formation rates to mass 
outflow rates (canonical wisdom suggests they are approximately equal), we find that $<40 \%$ of wind energy is radiated as Lyo.

4) Constraints on cosmic web properties. Limits on extended diffuse emission from cross-correlation (QSO-statistical, filament-statistical) are $\sim 1000 \mathrm{LU}$ [Table $2 \mathrm{R} 7, \mathrm{R} 9]$. Ly $\alpha$ filaments with filling factor $\mathrm{f}_{\mathrm{IGM}}=1$ are predicted to be $\sim 10-100$ LU, implying $\mathrm{f}_{\mathrm{IGM}}>0.01-0.1$.
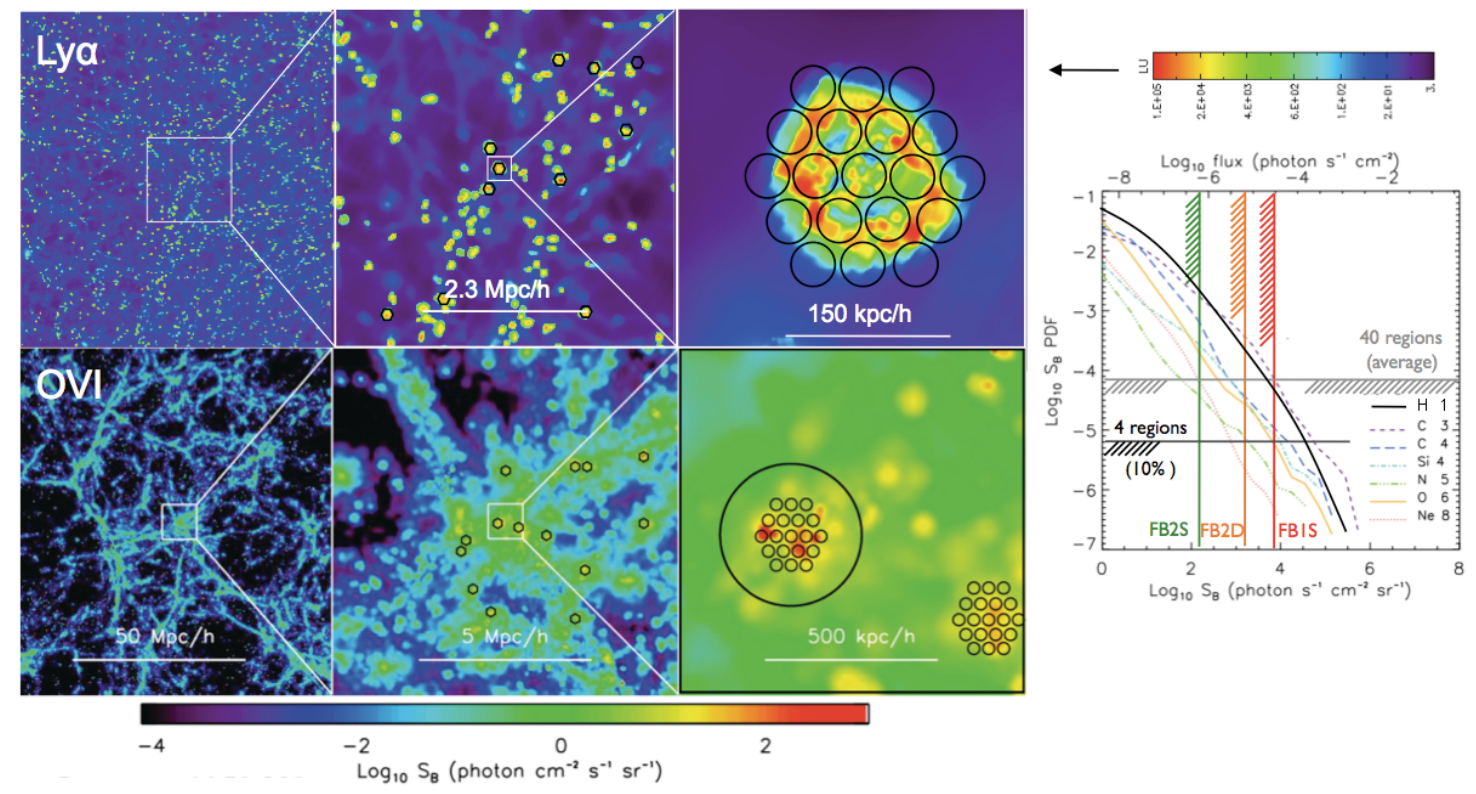

Figure 14. FIREBALL Gen-2 will detect metal and Ly $\alpha$ line emission from many CGM regions predicted by current IGM models. LOWER LEFT: Metal line simulations from Bertone et al. 2010 ${ }^{[5]}$ compared to FIREBALL-2. LEFT: shows images of a $27 \mathrm{Mpc}$ deep slice of OVI at $z=0.25$. CIV at $z=0.33$ is similar and somewhat brighter. Individual CGM mini-IFU bundles shown in middle panel, and single 19-fiber mini-IFU (50" diameter, 200-400 kpc) shown in right panel. UPPER LEFT: Same for Ly $\alpha$ simulations from our BINGO collaboration. RIGHT: Probability Distribution Function of CGM regions vs. Intensity for various emission lines. Vertical lines -- FB1S: FIREBALL statistical detection limit, FB2D: FB-2 direct limit, FB2S statistical limit. Horizontal lines -- PDF level where 40 regions are detected in $F B-2$ detection volume, and for 4 regions in the brightest $10 \%$ percentile, based on galaxy and CGM region volume density. Flux to the right and above these lines is detectable. In particular, FB-2-direct can detect CIII977, CIV1549, and OVI1033 at targeted redshifts in several bright regions.

\section{FIREBALL GEN-2}

FIREBALL-2 will achieve a dramatic increase in sensitivity by combining photon-counting, low-noise, UV-optimized CCDs (Gen-2 detectors) ${ }^{[8-10]}$ with a multi-IFU approach that targets bright Circum-Galactic Medium (CGM) regions. Sensitivity for direct detection of individual objects will be comfortably in the range predicted for Ly $\alpha$ and at the high end for metal lines (CIV1549, CIII977, and OVI1033, Figure 14) in CGM region ${ }^{[6]}$. FIREBALL Gen-2 observations are very likely to detect Ly $\alpha$ directly and CIV, CIII, and OVI either directly or statistically. In particular, the Bertone et al. ${ }^{[6]}$ models make a range of predictions dependent on the input physics, most notably various prescriptions for feedback energy input to and chemical enrichment of the CGM. FIREBALL Gen-2 measurements will constrain this poorly measured input physics that governs the formation and evolution of baryons in dark matter halos and the galaxies formed therein. For example, while detection of nearly all of the metal line CGM targets may provide strong support for present models, a lower detection rate could suggest that AGN feedback is working pervasively to suppress star formation and lower the metallicity of the IGM. 
Gen-2 Detectors. We are currently testing prototype Gen-2 devices based on commercial parts (e2v L3CCD CCD97) ${ }^{[8-}$ ${ }^{10]}$, and developing electronics that will lead to a flight package. We plan to design and manufacture 2 devices using CCD201 (each 1024 x $204813 \mu \mathrm{m}$ pixels). These will be thinned, Delta-doped, and AR-coated by JPL in the Microdevices Laboratory. We will update one of the laboratory L3CCD controllers to flight and modify the existing detector computer to support the new controller and telemetry format.

New IFU. We will manufacture a new set of 0.5-meter long multi-IFU bundles (two 20 mini-bundle units, one IFU plus spares). We will continue to use the in-house process developed for Flight 1 (though we will also consider some commercial options). For FIREBALL Gen-2, the polished fiber mini-IFUs will be inserted into a single focal plane plug plate mask (25 $\mathrm{mm}$ diameter). To correct for field rotation, the mask will be placed on a small rotational stage (rotation $<30$ degrees). We will also install a sliding shutter to select which of the 3 slits in the spectrograph focal plane will illuminate the detector. This shutter will also be used for dark calibration measurements. Although this bundle complement contains three times more fibers than what we have flown previously, the removal of the fiber vacuum feedthrough means that the task will be much more straightforward. We have significant experience with manufacture on this scale; for FIREBALL Gen-1 flights 1 and 2, Columbia manufactured 6 IFU bundles in total.

\section{ACKNOWLEDGEMENTS}

The material is based upon work supported by NASA under award No. NNX08AO39G. The FIREBALL collaboration also acknowledges considerable support from CNES, LAM, and CNRS. The FIREBALL flights would not be possible without the many contributions of the Columbia Scientific Ballooning Facility.

\section{REFERENCES}

[1] Rossin et al., "Semi-kinematic mount of the FIREBALL large optics", in [Proc. SPIE], 7018 (55), (2008).

[2] Tuttle, S. E., Schiminovich, D., Grange, R., Rahman, S., Matuszewski, M., Milliard, B., Deharveng, J.-M., and Martin, D. C., "FIREBALL: The first ultraviolet fiber fed spectrograph" in [Proc. SPIE], 7732 (78) (2010).

[3] Jelinsky, P. N., Morrissey, P. F., Malloy, J. M., Jelinsky, S. R., Siegmund, O. H. W., Martin, C., Schiminovich, D., Forster, K., Wyder, T., and Friedman, P. G., "Performance results of the GALEX cross delay line detectors," in [Society of Photo-Optical Instrumentation Engineers (SPIE) Conference Series], J. C. Blades \& O. H. W. Siegmund, ed., Society of Photo-Optical Instrumentation Engineers (SPIE) Conference Series 4854, 233-240 (Feb. 2003).

[4] Matuszewski, M., Evrard, J., Mirc, F., Milliard, B., Tuttle, S. E., Rahman, S., Martin, D. C., Schiminovich, D., Frank, S., McLean, R., and Chave, R., "FIREBALL: Instrument pointing and aspect reconstruction," in [Proc. SPIE], 7732(80) (2010).

[5] Rahman, S., Matuszewski, M., Tuttle, S. E., Milliard, B., Schiminovich, D., Martin, D. C., Frank, S., Evrard, J., Vibert, D., and Mirc, F., "FIREALL: Detector, data acquisition and reduction," in [Proc. SPIE], 7732(79) (2010).

[6] Bertone, S., et al., Metal-line emission from the warm-hot intergalactic medium: II. Ultraviolet, in arXiv. 2010. [B10].

[7] Dekel, A. and Y. Birnboim, Galaxy bimodality due to cold flows and shock heating, in Monthly Notices of the Royal Astronomical Society. 2006. p. 2.

[8] Morrissey, P., Martin, D. Christopher, Nikzad, S., Lingner, N. R., Schminovich, D., Hamden, E., "Ultraviolet Detectors for low surface brightness astronomy," in [Proc. SPIE], 7732(03) (2010).

[9] Jacquot, B. C., Monacos, S. P., Jones, T. J., Blacksberg, J., Hoenk, M. E., Nikzad, S., "Characterization and absolute QE measurements of delta-doped N-channel and P-channel silicon-based imaging arrays," in [Proc. SPIE], 7742 (18), (2010).

[10] Nikzad, S., Carver, A., Hoenk, M. E., Greer, F., "High-throughput processes for high-performance silicon imagers," in [Proc. SPIE], 7742 (48). 